Cahiers de recherches médiévales

Journal of medieval studies

$4 \mid 1997$

Être père à la fin du Moyen Âge

\title{
La manche et le cheval comme présents amoureux dans le Roman d'Alexandre d'Alexandre de Paris
}

\section{Bénédicte Milland-Bove}

\section{(2) OpenEdition \\ 12 Journals}

Édition électronique

URL : https://journals.openedition.org/crm/949

DOI : $10.4000 / \mathrm{crm} .949$

ISSN : 1955-2424

Éditeur

Honoré Champion

Édition imprimée

Date de publication : 15 décembre 1997

ISSN : 1272-9752

Référence électronique

Bénédicte Milland-Bove, "La manche et le cheval comme présents amoureux dans le Roman d'Alexandre d'Alexandre de Paris ", Cahiers de recherches médiévales [En ligne], 4 | 1997, mis en ligne le 15 janvier 2007, consulté le 15 décembre 2022. URL : http://journals.openedition.org/crm/949 ; DOI : https://doi.org/10.4000/crm.949

Ce document a été généré automatiquement le 15 décembre 2022.

Tous droits réservés 


\title{
La manche et le cheval comme présents amoureux dans le Roman d'Alexandre d'Alexandre de Paris
}

\author{
Bénédicte Milland-Bove
}

1 Le peu de place que tient la thématique amoureuse dans le Roman d'Alexandre est un des traits qui contribue à le distinguer de la production dite «romanesque» de la même époque. En effet, les romans antiques, qui s'élaborent dès le milieu du XII ${ }^{\mathrm{e}}$ siècle ${ }^{1}$, commencent à mêler à la matière guerrière des éléments témoignant d'une place nouvelle réservée aux figures féminines et à l'amour. Alexandre de Paris, lui, n'introduit que peu d'intermèdes amoureux et ceux-ci sont traités d'une manière bien peu courtoise ${ }^{2}$.

2 Dans ces conditions, le lecteur attentif peut être surpris par la présence de formules qui établissent, à l'échelle d'un vers, au beau milieu des récits de combats, un lien fugitif entre amour et prouesse. Ces deux motifs mettant en jeu des êtres de sexe féminin sont ceux du cheval et/ou de la manche comme présents amoureux, qui sont réunis dans l'exemple suivant:

Bos, li rois de Cartage, a sa gent amenee,

Et furent bien vint mil de noire gent barbee.

Il sist el cheval noir que li tramist la fee

Por cui amor passa un bras de mer salee ;

Une enseigne de paile a sa lance ot fremee (III, vv. 1838-1843). ${ }^{3}$

3 Ces formules, que l'on relève sous des formes proches dans les passages des branches II et $\mathrm{III}^{4}$ où l'influence de la chanson de geste est la plus grande, pourraient presque apparaître comme des motifs rhétoriques épiques. Cependant, l'équation qu'elles mettent en place relève plus de l'idéologie romanesque et courtoise que de celle de la chanson de geste. On les trouve d'ailleurs, de manière plus ou moins développée, dans les romans antiques. Mais l'usage spécifique qu'en fait le Roman d'Alexandre témoigne de la recherche d'une troisième voie, originale par rapport aux deux modèles invoqués. 
4 Les passages qui mentionnent le cheval et l'enseigne comme présents amoureux offrent de nombreuses ressemblances structurelles avec un ensemble plus vaste de formules sans aucun doute d'origine épique. Ces motifs rhétoriques, indexés par J.P. Martin dans son ouvrage sur les Motifs dans la chanson de geste ${ }^{5}$, peuvent apparaître dans deux types de contextes narratifs: soit dans le cadre statique d'un motif descriptif, appelé par J.P. Martin "histoire ou description des armes et des chevaux", soit dans le cadre dynamique du motif racontant «armement: chauces, hauberc, elme, espee, cheval, escu, espiel $»^{6}$. Selon les cas, on trouvera donc deux types de verbes introducteurs : «il sist », « il ert » « a sa lance ot »; ou « il monta », « il prist »....

5 Le motif de l'armement est parfois développé sur une dizaine de vers ${ }^{7}$. En revanche, les formules consacrées à l'origine des armes ou du cheval sont beaucoup plus brèves, et ce vraisemblablement à cause de leur position narrative, et de leur subordination étroite au récit de combat engagé. Elles interviennent le plus souvent en début de laisse, car elles permettent de passer du plan de la description générale de la bataille à la mise en valeur d'un combattant particulier ou de reporter l'attention du récit d'un combattant à un autre. Cette fonction de transition s'effectue la plupart du temps en évoquant de nouveau l'origine du guerrier (même lorsque celle-ci est bien connue), et en décrivant ses armes ou son cheval. Ainsi, à la branche III du Roman d'Alexandre, les laisses 111 à 113 utilisent ce type d'embrayeur narratif :

Litonas sist armés deseur un cheval noir Que li rois Alixandres li dona l'autre soir,

Gos li rois d'Outremons vit seur le brun seoir... (III, vv. 1908-1910)

Filotes sist armés el cheval espanois

Qui fu destre comés et si blans comme nois

Et ot en sa lance un gonfanon turquois (III, vv. 1925-1927).

Desor un destrier vair Perdicas sist armés,

Et vit un duc de Bastre qui de l'ost ert sevrés (III, vv. 1946-1947).

Ce type de formule est déjà présent dans la Chanson de Roland:

Li quens Gerins set el ceval Sorel

E sis cumpainz Gerers en Passecerf (vv. 1379-1380).

Elles peuvent mentionner aussi les qualités du destrier (le plus souvent sa rapidité), ou préciser son origine ${ }^{8}$ :

« Li arcevesque cumencet la bataille.

Siet el cheval qu'il tolit a Grossaille,

Ço ert uns reis qu'il ocist en Denemarche » (vv. 1487-1489).

Quant au motif de l'enseigne, on le trouve dans cette description de Roland:

As porz d'Espaigne en est passet Rollant

Sur Veillantif, sun bun cheval curant.

Portet ses armes, mult li sunt avenanz,

Mais sun espiet vait li bers palmeiant,

Cuntre le ciel vait la mure turnant,

Laciet en su un gunfanon tut blanc;

Les renges li batent josqu'as mains (vv. 1152-1158).

9 Les «variantes » précisant l'origine amoureuse de ces attributs n'apparaissent pas dans la Chanson de Roland ni dans les autres chansons de geste les plus anciennes telles Gormont et Isembart, ou la Chanson de Guillaume... J. P. Martin ne les mentionne pas non plus, car elles n'apparaissent pas en tant que telles dans son corpus'. Il serait tentant d'en conclure que les romans antiques ont construit de nouveaux motifs romanesques, 
sur le modèle des motifs épiques, pour mieux introduire, au cœur même des épisodes guerriers, la thématique amoureuse. Mais le jeu d'échange entre les deux formes au XII siècle est complexe. Assez vite, on les trouve sous des formes proches dans des chansons un peu plus tardives, témoignant de l'influence de la littérature romanesque et courtoise. Ainsi, dans Girart de Vienne, c'est le motif du don de l'enseigne qui est utilisé : Aude, à la demande de son frère, fait don d'un magnifique gonfanon à Lambert, ce qui ne manque pas d'exciter la jalousie de Roland ${ }^{10}$. Dans les Enfances Guillaume ${ }^{11}$, le héros s'empare du cheval Balzan destiné par Orable à son fiancé Thibault. Ce rapt préfigure le détournement de l'amour de la belle sarrasine en faveur du héros chrétien, ce qui n'est pas sans rappeler l'histoire de Diomède et Briséïda.

Dans les romans antiques, les occurrences des motifs du don de la manche et du cheval sont de deux ordres. Les premières se repèrent dans des passages où l'influence des motifs épiques reste prédominante. C'est bien dans le contexte narratif de l'armement que, dans le Roman de Thèbes ${ }^{12}$, l'origine du cheval d'Éthéocle est par deux fois indiquée :

Puis li ameinent Blanchenue

Que li tramist l'autre an sa drue ;

Galathea ot non s'amie,

si fu fille au roi de Nubie (vv. 6241-6245).

Un jour a fet li rois s'oissue,

si fu montez sus Blanchenue,

que li tramist l'autr'ier s'amie ;

Mout est bons pour chevalerie.

Hiaume porta, escu et lance (vv. 8595-8699).

11 Il en va de même pour la mention de la manche que lui a donnée une deuxième amie, Salemandre, la fille de Daire :

son escu prist, l'iaume laça ;

derriere ot une manche vaire

que li tramist la fille Daire (vv. 8484-8486).

Dans le Roman de Troie ${ }^{13}$, la mention de Galatée, cheval donné à Hector par la fée Orva, apparaît dans le même environnement narratif :

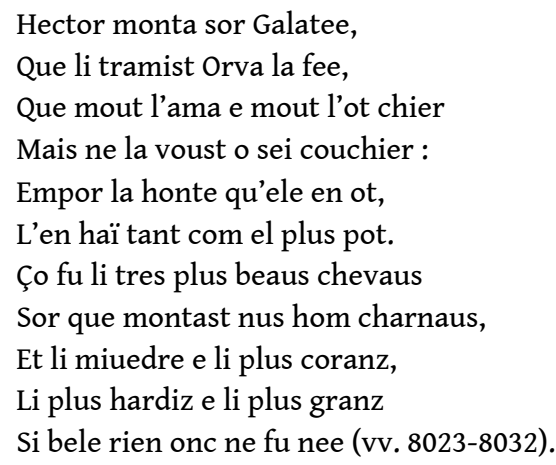

On voit cependant que cette mention du cadeau de la fée est suivie d'un développement sur les relations entre Orva et Hector, qui est déjà une amorce d'amplificatio romanesque destinée à doubler la réputation guerrière d'Hector d'une aura amoureuse. Plus loin dans le roman, la présentation de Celidis, jeune grec ami de la reine de Femenie qui lui a donné son cheval et ses armes, constitue également un portrait plus romanesque qu'épique : 
Reis Celidis esteit moult beaus,

Lons, grailes, dreiz, juevnes toseaus.

La reïne de Femenie

Aveit esté lonc tens s'amie :

Por li esteit mout essauciez,

Mout coneüz e mout preisiez.

Ses armes e son milsoudor,

de chierté e de fine amor,

Li ot tramis, s'en ert ermez

Por ço ert sovent remirez (vv. 8832-8841).

14 Les romans antiques, tout en reprenant lors des descriptions de bataille un mode d'écriture proche de l'esthétique épique, introduisent des modifications qui sont autant d'adaptations aux thématiques et aux techniques romanesques qu'ils mettent en place. Les motifs de la manche et du cheval sont intégrés à des passages témoignant de techniques narratives nouvelles et d'une dramatisation plus ou moins poussée et réussie selon les romans.

Ainsi, dans le Roman de Thèbes, on mentionne le don d'une manche fait par Ysmaine à Athon lors de la description physique de la jeune fille, insérée par le narrateur au moment où elle se rend en ambassade avec sa mère et sa sœur auprès de son frère Polynice :

Ysmaine fu amie Athon,

si ot vestue un siglaton.

La manche destre en ot sevree,

ele l'avoit Athon donnee (vv. 4089-4092).

Plus loin, c'est grâce à cette manche que, depuis les créneaux d'où elle contemple la bataille, la jeune fille peut reconnaître Athon, qui vient de réussir un beau coup ${ }^{14}$. Le regard féminin, dans les trois romans, acquiert donc une importance nouvelle. Les femmes observent et commentent la bataille. L'enjeu, pour les hommes comme pour les femmes, est de se faire voir, d'où l'importance de l'enseigne comme signe visuel chargé de manifester à tous, et à l'être aimé en particulier, la relation amoureuse et courtoise.

Dans le Roman d'Énéas ${ }^{15}$, le motif de la manche est évoqué sur le mode de l'irréel du passé et intégré cette fois au sein d'un monologue intérieur de Lavine. La jeune fille, montée elle aussi en haut d'une tour pour regarder le combat singulier entre Énée et Turnus, regrette de ne pas avoir, pour encourager le héros, pensé à lui envoyer ce gage :

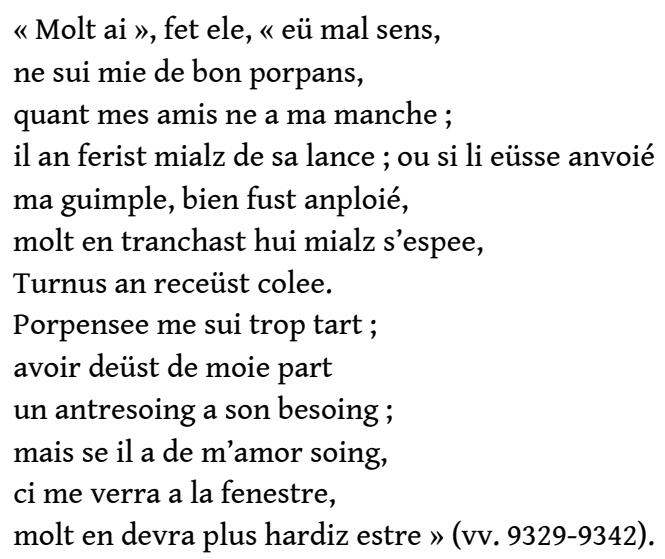

Le motif est donc ici réutilisé selon un mode totalement romanesque, puisqu'il s'insère dans l'évocation des tourments, des hésitations, des atermoiements provoqués par l'amour chez les héros. 
19 Mais c'est Benoît de Sainte-Maure qui, lors de l'épisode des amours de Briséïda, utilise ces motifs comme de véritables ressorts romanesques, participant à la progression dramatique. Les échanges qui se font entre ces gages symbolisent le changement des sentiments de la jeune fille. Ainsi, lors de la huitième bataille, Diomède désarçonne Troïlus et s'empresse d'envoyer son destrier à la jeune fille ${ }^{16}$, qui accepte le présent, mais sans paraitre en savoir meilleur gré au jeune chef grec. Toujours lors de cette même bataille, et lors d'une occurrence relevant d'une "transition» épique, Benoît mentionne que Troïlus porte sur sa lance l'enseigne de son amie :

Li confanons cui li dona

Dameisele Briseïda,

Que mout l'amot ancore al jor (vv. 14449-14451).

C'est en reprenant avec art ces deux motifs que Benoît matérialise le nouveau choix de Briséïda. Un jour, Diomède trouve Briséïda en train de contempler le cheval de Troïlus ${ }^{17}$. L'écriture de la scène, et le discours de l'héroïne elle-même, sont d'une habileté consommée. Benoît laisse planer un doute sur les sentiments de l'héroïne : songe-t-elle à Troïlus? C'est ce que laisse penser l'indication qu'il donne immédiatement après. Le désir qu'a éprouvé la jeune fille de pouvoir renvoyer sa monture au Troyen aurait bien sûr constitué un désaveu pour Diomède. Ensuite elle s'adresse à Diomède d'un ton moqueur, mêlant habilement dans ses propos le chaud et le froid, les provocations et les encouragements. Diomède a eu tort de lui donner le cheval puisqu'il a ensuite été désarçonné; elle propose de le lui prêter et le défie de réussir à le défendre jusqu'au bout contre Troïlus. Diomède accepte sans s'y tromper : il s'agit là pour Briséïda d'une manière d'agréer son amour. Le don que lui fait ensuite la jeune fille d'une manche vient parachever ce retournement d'alliances :

La destre manche de son braz

Nueve e fresche d'un ciglaton

Li baille en lieu de confanon (vv. 15176-15178).

21 Le lecteur est invité à mettre en parallèle Troïlus dont le lien amoureux n'est plus exprimé qu'unilatéralement, dans des passages relevant des stéréotypes guerriers, et Diomède, qui reçoit avec tout l'éclat de la manche neuve les honneurs du nouveau duo amoureux.

Le Roman de Troie témoigne de l'aboutissement du travail d'appropriation et de transformation d'un cliché qui au départ révélait encore des liens avec une technique épique, mais qui se signale désormais comme l'ébauche en raccourci de la nouvelle formule romanesque, promise à un brillant avenir, entrelaçant les armes et l'amour. À la différence de ce qui se passera ensuite pour le roman arthurien plus tardif, l'impulsion narrative des romans antiques reste indépendante de la motivation amoureuse. L'équation entre amour et prouesse a un statut ornemental et non vital dans l'engendrement du récit ${ }^{18}$. Ces chevaliers, qui combattent avant tout pour prendre ou défendre Thèbes, Troie ou Laurente, se battent aussi et de surcroît pour leur amie, et leur désir de s'illustrer se trouve renforcé par celui de briller à leurs yeux. Aussi, c'est toute une armée arborant des manches en guise d'enseigne que nous présente Benoît :

Tant i perent heaume d'acier,

Tant bel escu, tant bon destrier

E tante enseigne despleiee,

Tante bele arme entreseigniee

Et tante manche de bliauz

D'orfreis, de paile e de cendauz (vv. 8321-8326). 
Dans ces formules, c'est donc bien la nouvelle saveur romanesque qui se déguste à l'état de concentré narratif. On peut en mesurer l'audace si l'on se rappelle que le cheval, insigne par excellence de la fonction guerrière, est habituellement avant tout un cadeau du souverain. De même, porter la manche de son amie, c'est substituer ses couleurs à celles de son suzerain, ou du moins les mettre à égalité. Or il semble au contraire que c'est bien la suprématie de la figure royale que le Roman d'Alexandre veut consacrer par l'utilisation spécifique de ces motifs.

Face à ces innovations, l'utilisation que fait le Roman d'Alexandre de ces formules paraît singulièrement marginale et traditionnelle. Pourquoi avoir repris ce motif courtois d'une manière aussi peu romanesque, en le replaçant au contraire dans un cadre strictement épique?

En effet, le Roman d'Alexandre n'utilise ces motifs que dans les passages où l'influence de l'écriture épique est la plus grande, à savoir les récits de bataille. Ces formules n'apparaissent pas toujours en début de laisse mais permettent d'introduire les protagonistes de l'affrontement qui va se dérouler. À la différence des romans antiques, qui les intégraient parfois au récit de l'armement, le Roman d'Alexandre ne les insère qu'en pleine relation de combat, ce qui, bien sûr, limite les digressions possibles. Elles ne donnent donc lieu à aucune amplification romanesque du type de celles qu'on a rencontrées dans les romans antiques. Autre différence : elles mettent en jeu des êtres féminins anonymes, ne jouant aucun rôle dans l'action, et dont c'est la seule mention. Elles concernent d'ailleurs la plupart du temps des comparses, chevaliers ennemis des Grecs. Alexandre lui-même n'est jamais impliqué. L'unique fois où il se bat pour une femme, pour récupérer l'épouse du fils de Candace ${ }^{19}$, il agit pour un autre et sous une autre identité. Si, à la fin du roman, la Reine des Amazones lui fait don de son cheval ${ }^{20}$, animal merveilleux qu'il a admiré lors du tournoi donné en son honneur, on ne le voit jamais combattre sur cette monture, puisque la mort vient mettre un terme à ses conquêtes.

Il est frappant que le motif du cheval comme présent amoureux n'apparaisse que pour des chevaliers appartenant à l'armée ennemie d'Alexandre. Lors de l'épisode du «Fuerre de Gadre », Dilas coupe la tête d'un baron de Grèce :

Mais Dilas l'orguelleus, qui siet sor Noirenue, -

Un destrier de grant pris que li dona sa drue,

Armés fu et couvers d'une porpre volsue... (II, vv. 2282-2284).

27 À la branche III, c'est Astarot de Nubie qui monte un cheval donné par une fée :

Ançois que il eüst sa raison definee,

Astaros de Nubie a l'angarde montee,-

Et sist el cheval noir que li dona la fee,

C'estoit une pucele qu'il avoit molt amee,

Por cui amor passa un bras de mer salee (III, vv. 1741-1745).

Quelques centaines de vers plus loin, on trouve presque exactement la même formule :

Bos, li rois de Cartage, a sa gent amenee,

Et furent bien vint mil de noire gent barbee.

Il sist el cheval noir que li tramist la fee

Por cui amor passa un bras de mer salee ;

Une ensegne de paile a sa lance ot fremee. (III, vv. 1837-1842). 
Dans tous ces exemples, les chevaliers sont peu après abattus par l'un des douze pairs : Dilas est désarçonné par Clin qui s'empare du cheval, c'est aussi Clin qui vient à bout d'Astarot, et Alexandre lui-même abat le roi de Carthage.

De plus, ce motif prend une signification particulière de par sa répartition singulière et l'opposition avec ce qui caractérise les lieutenants d'Alexandre. D'un côté, on a des combattants orientaux dont le cheval est un don de l'amour, de l'autre les chevaliers de l'armée d'Alexandre pour qui le cheval provient toujours de la largesse de leur empereur. Quelques exemples parmi d'autres :

Emenidus fu preus et fu nes de Valterne,

N'ot millor chevalier trusqu'en fine posterne.

Il sist el cheval noir que on claime Pïerne,

Sel tramist Alixandre uns princes de Salerne (III, vv. 693-696).

Litonas sist armés deseur un cheval noir

Que li rois Alixandre li dona l'autre soir (III, vv. 1908-1909).

La répartition du motif de la manche semble la même, quoique un peu moins nette. Sur les quatre occurrences, deux concernent des chevaliers orientaux, qui sont finalement vaincus. Lors de la bataille contre le duc Bétys, Gadifer, un de ses lieutenants, est paré de cet ornement galant :

Gadifer vint molt tost, qu'il n'a talent qu'il rie ;

En son bras ot lacie une manche s'amie,

Qui n'estoit mie garce ni pauvrement norrie,

Mais riche damoisele, fille au roi d'Aumarie.

Si fiert Emenidus en la targe florie. (II, vv. 1498-1502).

À la laisse 118, un autre roi, Aminadap porte à son bras droit la manche de son amie :

Devant toute sa gent requiert chevalerie,

Et porte en son bras destre une manche s'amie ;

Qant li solaus i fiert, bien luist et reflambie » (III, vv. 2041-2043).

Mais, à la laisse 122, Aminadap captif a perdu sa belle manche brodée :

Aminadap fu pris et li orfrois perdus. (III, v. 2109)

Deux autres occurrences concernent cette fois des lieutenants d'Alexandre, Émenidus et Tholomé. À la laisse 376, Tholomé se prépare au combat :

L'ensegne de sa lance li tramist par amor

La roïne d'Égypte, qui puis l'ot a segnor (III, vv. 6428-6429).

À première vue, ceci semble contredire la répartition que nous essayons de dégager. Mais ce détail prend tout son sens à la branche IV, où l'on apprend que cette union avec la reine d'Égypte est un mariage arrangé par le roi de Macédoine: on retrouve làderrière la générosité d'Alexandre, qui fait don de l'Égypte à son fidèle compagnon ${ }^{21}$.

Il y a donc dans le texte des rappels de l'opinion commune, d'un certain type d'explication de la prouesse, mis en avant par toute la littérature romanesque courtoise $\mathrm{e}^{22}$. Mais, en l'attribuant systématiquement aux ennemis vaincus, en y associant une causalité magique (puisque les pucelles sont parfois désignées comme fées) et de plus inopérante, l'auteur disqualifie ce fonctionnement. Il lui oppose un autre moteur, qui, lui, est efficace. L'amour, c'est bon pour les lais bretons et pour ces chevaliers amollis que sont les Orientaux, mais ce qui motive et fait avancer l'armée conquérante, c'est la largesse d'Alexandre! G. Duby, dans Le Dimanche de Bouvines, s'étonne de la présence, complètement marginale dans le récit de la bataille fait par Guillaume le Breton, de l'exclamation attribuée au flamand Jean Buridan «Que chacun pense à sa 
belle! ». Il y voit une manière de dénoncer la légèreté de l'ennemi, de le discréditer ${ }^{23}$. On aurait ici la même intention, Alexandre de Paris se contentant de suggérer par ces formules ce que le dialogue suivant entre Astarot de Nubie et Clin dit de manière beaucoup plus claire :

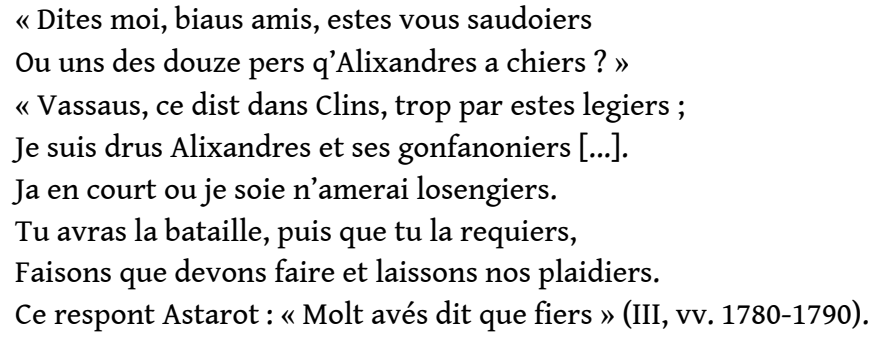

37 Le rapprochement à la rime à quelques vers d'intervalle des deux adjectifs «fiers » et «legers» souligne nettement le contraste entre Astarot, qui se répand en bavardages courtois avant la bataille, et Clin, qui n'a que mépris pour ces frivolités.

Il ne faut cependant pas voir dans cette mise à distance un discrédit total porté sur les relations amoureuses. On trouve en effet malgré tout un exemple qui s'applique à Émenidus :

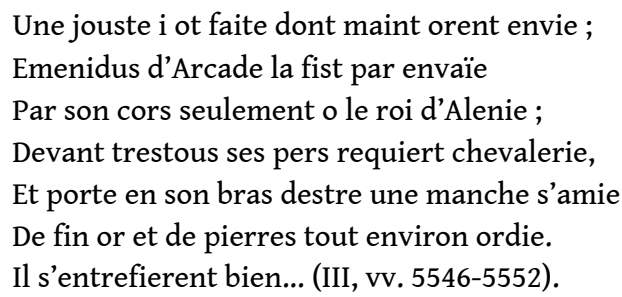

39 À la différence d'Aminadap ou des autres chevaliers orientaux, Émenidus, lui, est capable de conserver cette manche qui se distingue par son exceptionnelle richesse. À la branche IV, c'est encore à propos d'Émenidus que l'on trouve une autre mention surprenante du lien entre amour et prouesse :

Emenidus, li fieus l'amiraut de Tudele,

Qui por l'amor avoir de la noble pucele

Combati au gaiant es plaines de Castele,

Tant que le cuer li traist par desous la mamele (IV, vv. 722-725).

Le lecteur semble ici comme transporté dans un autre univers, avec un motif qui rappelle plus les légendes celtiques ou arthuriennes que l'univers épique. Ces formules n'assument-elles donc pas avant tout une fonction d'ouverture de la laisse et de l'œuvre tout entière, en ménageant une échappée vers un autre domaine narratif ? À la branche IV, Alexandre de Paris mentionne parmi son public des dames et des pucelles, dans une formule qui évoque le guerredon du service amoureux ${ }^{24}$. Il s'agirait tout simplement de clins d'oeil à fonction phatique destinés à une partie de son auditoire, pour lui montrer qu'il n'ignore pas les nouveautés littéraires, qu'il serait capable de les exploiter, mais qu'il a volontairement cherché ailleurs une autre recette narrative.

41 La présence dans le Roman d'Alexandre de ces formules mentionnant le cheval ou l'enseigne comme présents amoureux est donc à tous égards déroutante. Marginales, elles font intervenir des êtres liés à d'autres traditions littéraires, ainsi qu'une conception des liens entre hommes et femmes plus «romanesque» qu'épique. Impliquant dans une occurrence isolée le lieutenant préféré d'Alexandre, Émenidus, par surcroît son gonfalonier, elles résistent à une interprétation univoque, purement 
idéologique, selon laquelle elles seraient destinées (en réaction contre le rôle nouveau dévolu à l'amour) à valoriser la largesse de la figure royale comme unique source de la valeur de son armée.

Traces d'une influence des romans antiques qui, les premiers, ont greffé sur la matière épique des épisodes amoureux et ont utilisé ces formules dans les passages où ils expérimentaient de nouvelles techniques d'écriture (portraits, monologues amoureux, dialogues et récits linéaires dramatiques qui ne sont plus brisés par les retours au même des laisses), elles sont au contraire présentes dans le Roman d'Alexandre dans les vers qui se conforment le plus aux canons de la chanson de geste.

Assumant sans doute une fonction phatique, destinées à frapper le public par leur incongruité et leur rareté mêmes, elles l'invitent à mesurer la réussite de ce récit qui se passe de l'inventio et de l'amplificatio de la thématique amoureuse, et met à sa source le désir de savoir et de découvrir. Comme son héros éponyme qui, à force de s'aventurer dans des contrées toujours plus lointaines et toujours plus inconnues, finit par se heurter au monde arthurien en rejoignant les Bornes Artu, Alexandre de Paris s'autorise de brèves échappées qui rapprochent de son univers narratif ceux qu'il a délibérément laissés de côté.

\section{NOTES}

1. On date le Roman d'Alexandre des années 1180. Le Roman de Thèbes a été écrit vers 1150, l'Enéas vers 1160 et le Roman de Troie de Benoît de Sainte-Maure vers 1165. 2. Voir la thèse de C. Gaullier-Bougassas Le Roman d'Alexandre d'Alexandre de Paris et le Roman de toute chevalerie de Thomas de Kent : frontières de l'épique et du romanesque, Paris III, 1995, et son article "Alexandre et Candace dans le Roman d'Alexandre d'Alexandre de Paris et le Roman de toute chevalerie de Thomas de Kent ", Romania t. 112, 1991, pp. 18-44. 3. The Medieval French Roman d'Alexandre, vol. II (Version of Alexandre de Paris), edited by E.C. Armstrong, D.L. Buffum, B. Edwards, L.F.H. Lowe, Princeton, 1937, Elliot Monographs 37, reed. New York, Kraus Reprints, 1965.

4. Branche II vv. 1499-1501; 2282-2283 ; br. III vv. 1743-1745, 1840-1841; 2042-2043 ; $5549-5550 ; 6428-6429$.

5. Les motifs dans la chanson de geste, Centre d'études médiévales et dialectales de l'université de Lille III, 1992.

6. Ibid., pp. 358-359. J.P. Martin inclut dans le motif de «l'armement» le motif du " chevalier armé », qui est bien une description canonique et non un "motif traduisant une action " (définition du motif racontant).

7. Voir par exemple la laisse LXXIX de la Chanson de Roland, vv. 994-1004, qui décrit collectivement l'armement de l'armée païenne et mentionne les « gunfanons blancs e blois et vermeilz » (v. 999) et les « destrers» (v. 1001). La Chanson de Roland, éd. J. Dufournet, Paris, Garnier-Flammarion, 1993.

8. Voir l'article de J. Frappier, "Les destriers et leurs épithètes ", Congrès et colloques de l'université de Liège, vol. 11, 1959. A. Micha, dans son article sur la «Couleur épique dans 
le Roman de Thèbes ", donne une liste complète des formules se rapportant aux chevaux dans ce roman (Romania, t. 91, 1970, p. 150).

9. Dans Gerbert de Metz, la reine et sa fille, toutes deux amoureuses du héros, lui envoient, ainsi qu'à ses compagnons, des présents, dont un «bon destrier gascon ». Mais il s'agit d'un motif bien différent (celui de "la princesse amoureuse»), que J. P. Martin classe parmi les motifs modalisateurs. Il est développé pour lui-même et ne promeut en aucune façon l'alliance des armes et de l'amour. Le cheval, d'ailleurs, n'est pas destiné à Gerbert mais à Gerin, ce n'est donc pas à proprement parler un présent amoureux (Gerbert de Metz, chanson de geste du XII e siècle, éd. P. Taylor, Bibliothèque de la faculté de Philosophie de Namur, fasc.11, Namur-Lille-Louvain, 1952, vv. 3720-3726).

10. Girart de Vienne de Bertrand de Bar-sur-Aube, éd. W. van Emden, Paris, Picard, SATF, 1977. Voir vv. 3814-3857 ; 3874-3876 ; 3930-3931.

11. Les Enfances Guillaume, chanson de geste du XIII ${ }^{e}$ siècle, éd. P. Henry, Paris, SATF, Picard, 1935.

12. Le Roman de Thèbes, éd. G. Raynaud de Lage, Paris, Champion, CFMA, 1966 et 1968.

13. Le Roman de Troie en vers, éd. L. Constans, Paris, SATF, 1904-1912.

14. Le Roman de Thèbes, op.cit., vv. 5857-5909.

15. Le Roman d'Enéas, éd. J.J. Salverda de Grave, Paris, Champion, CFMA, 1973.

16. Déjà dans le Roman de Thèbes, Parthénopée envoie un cheval gagné à l'ennemi à Antigone et dit à son messager :

«Par ces enseingnes mant m'amie pour lui ai fet chevalerie » (Roman de Thèbes, op.cit, vv. 4591-4598).

Le don du cheval, dans les romans antiques, fonctionne donc bien dans les deux sens, comme gage et encouragement de la prouesse, ce qui n'est pas le cas dans le Roman d'Alexandre, où l'on ne voit jamais un chevalier offrir un destrier à sa belle.

17. Le Roman de Troie, op.cit., t. III, vv. 15009-15112.

18. On peut mesurer la différence si l'on songe par exemple à certains passages du Lancelot en prose, tel celui où Lancelot refuse de donner libre cours à sa vaillance et d'accomplir tous les exploits dont il est capable si Guenièvre ne l'y invite pas expressément. Sans la reine, sans ardeur amoureuse, pas de prouesse, et donc pas de récit. (Lancelot, roman en prose du XIII siècle, éd. A. Micha, Genève, Droz, 1982, t. VIII, LIIa, 30-37).

19. III, vV. 4514-4677.

20. III, vv. 7699-7705.

21. IV, v. 289 ; vv. 304-320.

22. Autre occurrence d'une remarque du même type, mais qui ne relève ni du motif du cheval ni du motif de l'enseigne, cette remarque de l'émir de Babylone, qui commente un beau coup réussi par Macabrun par ces mots :

«Par foi, dist l'amiraus, ceste joste est molt bele, Cist doit avoir amor et deduit de pucele »(III, vv. 7003-7004).

Là encore, Macabrun est vaincu par un Grec.

Pour un relevé de l'ensemble des passages établissant un lien entre amour et prouesse dans les romans antiques et dans le Roman d'Alexandre, on consultera A. Petit, Naissances du Roman. Les techniques littéraires dans les romans antiques du XII e siècle, Paris, Champion, 2 vol., 1985, pp. 338-341. A. Petit ne discute pas le cas du Roman d'Alexandre et interprète toutes ces occurrences comme autant de signes de la naissance d'une équation romanesque nouvelle.

23. G. Duby, Le dimanche de Bouvines, Paris, Gallimard, 1973, p. 32. 
24. «Li gentil chevalier et li clerc sage et bon, Les dames, les puceles, qui ont clere façon, Qui sevent de service rendre le guerredon, Cil doivent d'Alixandre escouter la chançon » (IV, vv. 1652-1655). 